\title{
miR-133a, directly targeted USP39, suppresses cell proliferation and predicts prognosis of gastric cancer
}

\author{
XIANG DONG $^{1 *}$, HAILONG SU ${ }^{2 *}$, FENG JIANG $^{3}$, HAIYAN LI $^{4}$, GUANGWEN SHI $^{5}$ and LIJUAN FAN ${ }^{1}$ \\ ${ }^{1}$ Digestive System Department, Jining First People's Hospital, Jining, Shandong 272011; ${ }^{2}$ Department of General Surgery, \\ Affiliated Yantai Yuhuangding Hospital of Qingdao University, Yantai, Shandong 264000; Departments of ${ }^{3}$ Imaging, \\ ${ }^{4}$ Anesthesiology and ${ }^{5}$ Obstetrics, The People's Hospital of Zhangqiu Area, Jinan, Shandong 250200, P.R. China
}

Received October 2, 2017; Accepted March 2, 2018

DOI: $10.3892 / \mathrm{ol} .2018 .8421$

\begin{abstract}
Gastric cancer has high incidence and mortality, and the mortality ranks second only to lung cancer. Downregulation of miR-133a has been observed in certain types of tumors, and it is involved in gastric cancer. The aim of the present study was to explore the molecular mechanisms of miR-133a and ubiquitin-specific protease 39 (USP39) in gastric cancer. Western blot analysis and RT-PCR were employed to measure miR-133a and USP39 expression. To confirm whether miR-133a targeted USP39, we conducted a luciferase reporter assay. We utilized 3-(4,5-dimethyl-2-thiazolyl)-2,5-diphenyl-2H-tetrazolium bromide (MTT) assay to detect the effects of miR-133a on gastric cell proliferation. miR-133a was significantly downregulated in cancer tissues and cell lines (HGC-27 and MGC-803), while the expression level of USP39 was higher in tumor tissues than in paracancerous tissues. Upregulated expression of miR-133a and/or USP39 downregulation could inhibit cell proliferation in gastric cancer cells. Furthermore, USP39 was identified as a direct target of miR-133a and the inverse relationship between them was also observed. USP39 was a firsthand target of miR-133a and there was a negative correlation between them. In addition, a low expression of miR-133a or overexpression of USP39 predicted poor prognosis. In conclusion, miR-133a may be a novel therapeutic target of microRNA-mediated suppression of cell proliferation in CC, but the role of the miR-133a/USP39 axis in CC progression needs further study.
\end{abstract}

\section{Introduction}

Gastric cancer has high mortality, ranks second only to lung cancer and the incidence is fourth with 400,000 individuals

Correspondence to: Dr Lijuan Fan, Digestive System Department, Jining First People's Hospital, 6 Jiankang Road, Jining, Shandong 272011, P.R. China

E-mail: jkol44048@126.com

${ }^{*}$ Contributed equally

Key words: miR-133a, gastric cancer, proliferation, prognosis, USP39 suffering gastric cancer annually in China, according to a global statistical report published by the World Health Organization (WHO) in 2016. Patients with gastric cancer were generally diagnosed at an advanced stage with poor prognosis and recurrence (1-4). Therefore, identifying tumor molecular markers for early diagnosis is essential for patients with gastric carcinoma.

MicroRNAs (miRNAs/miRs) are a group of small non-coding regulatory molecules with 22-28 nucleotides sequence in length (5). miRNAs through base pairing with the 3'-untranslated regions (3'UTR) of target messenger RNAs (mRNAs) or directly cleaving the mRNA at the post-transcriptional cause mRNA degradation and/or translational repression (6-9). miR-133a, a kind of miRNA, has been reported to be aberrantly expressed in several carcinomas, including osteosarcoma, ovarian carcinoma, lung cancer, esophageal carcinoma and even in gastric carcinoma and other types of cancer (10-13). In oral squamous cell carcinoma, miR-133a inhibits cell proliferation and invasion by suppressing COL1A1 (14). Furthermore, in colorectal cancer, miR-133a acts as a tumor suppressor to inhibit cell growth and migration by targeting elF4A1 in vitro (15). In addition, miR-133a had a low expression and inhibited cell proliferation, invasion and induced apoptosis in gastric carcinoma cells $(12,16,17)$. It could also bind to a variety of genes, including COL1A1, FSCN1 and ubiquitin specific protease 39 (USP39) $(14,16,18$ ). It has been reported that miR-133a inhibits cell progression by targeting USP39 in pancreatic cancer (18).

USP39, one of the deubiquitinating enzymes (DUBs) without ubiquitin protease activity, is involved in RNA splicing and was necessary to maintain a spindle checkpoint (19-22). USP39 has been demonstrated to act as an oncogenic factor in many tumors, such as colorectal cancer, lung cancer, melanoma, pancreatic cancer and osteosarcoma cancer $(18,23-26)$. In osteosarcoma cells, a decreased USP39 expression inhibited cell growth and induced apoptosis (26). In melanoma, knockdown USP39 inhibited cell growth and induced cell cycle arrest and apoptosis (25). Thus, to determine the molecular mechanism of miR-133a and USP39 on cell proliferation and prognosis is critical for planning therapeutic strategies, assessing prognosis, and monitoring response to therapy. Therefore, in the present study, we aim to test the functional role that miR-133a plays in tumor progression and 
the correlation with USP39. Furthermore, we also measured the overall survival (OS) and disease-free survival (DFS) according to the expression of miR-133a and USP39.

\section{Materials and methods}

Patients and clinical samples. According to WHO classification, we obtained 53 paired gastric cancer tissues and paracancerous tissues (PT) from patients presenting for treatment in Jining First People's Hospital (Jining, China) from January 2014 to December 2016. Fresh resected samples were immediately cut and snap-frozen in liquid nitrogen and stored in a freezer at $-80^{\circ} \mathrm{C}$. The patients had no treatment before surgery. The complete clinicopathological characteristics of patients, including age, sex, tumor grade and TNM stage are described in Table I. Informed consent to use the specimens in this study was obtained from the patients. The study was approved by the Ethics Committee of Jining First People's Hospital (Jining, China).

Cell lines and culture condition. Two gastric cancer cell lines (HGC-27 and MGC-803) and one normal gastric cell GES-1 were purchased from the American Type Culture Collection (Rockville, MD, USA). The cells were maintained in RPMI-1640 medium (Gibco, Carlsbad, CA, USA) add in 15\% fetal bovine serum (Sigma-Aldrich, St. Louis, MO, USA) cultured at $37^{\circ} \mathrm{C}$ in a fully humidified atmosphere containing $5 \% \mathrm{CO}_{2}$.

RNA isolation and RT- $q P C R$. Total miRNAs or mRNAs were isolated and purified by TRIzol reagent (Invitrogen, Carlsbad, CA, USA) or miRcute and Separation of miRNAs kit (Tiangen Biotech Co.,Ltd., Beijing, China). Initially, reverse transcription was employed to produce the first cDNA chain used PrimeScript $^{\mathrm{TM}}$ II 1st Strand cDNA Synthesis kit (Takara Biotechnology Co., Ltd., Dalian, China). Quantitative PCR was then carried out using SYBR Prime Script miRNA RT-PCR kit or SYBR Premix kit (both purchased from Takara Bio, Inc., Otsu, Japan) according to the manufacturer's protocol. The primer sequences used were: miR-133a forward, 5'-ATAAGAATGCGGCCGCATTCCAAACTAGCAGCA CTA-3' and reverse, 5'-AGCTTTGTTTAAACTTAACCATT CTAGCTTTTCC-3'; USP39 forward, 5'-GCTGATGATGAT TGATGCT-3' and reverse, 5'-GCTCCAAGAATCCCAAGG CT-3'; GAPDH forward, 5'-CCACTCCTCCACCTTTGAC-3' and reverse, 5'-ACCCTGTTGCTGTAGCCA-3'; and U6 forward, 5'-CTTCGGCAGCACATATACT-3' and reverse, 5'-AAAATATGGAACGCTTCACG-3'. The thermocycling parameters were as follows: $95^{\circ} \mathrm{C}$ for $3 \mathrm{~min}$ and 40 cycles of $95^{\circ} \mathrm{C}$ for $15 \mathrm{sec}$ followed by $60^{\circ} \mathrm{C}$ for $30 \mathrm{sec}$. The normalization of miRNA and mRNA was U6 and GAPDH, respectively. miRNA and mRNA expression levels were subsequently calculated using the $2^{-\Delta \Delta \mathrm{Cq}}$ method. All the RT-qPCRs were run in triplicate.

Transfection. For the miRNA, due to its downregulation of miR-133a in gastric cancer cells HGC-27 and MGC-803, miR-133a was overexpressed through transfected miR-133a mimic. In addition, USP39 was intervened used small interfering RNA (siRNA) to measure the efficiency of USP39 in gastric cancer cells.
Suitable cells were inoculated into 6-well plates and cultivated overnight at $37^{\circ} \mathrm{C}$. And then we transfected the special vector utilized Lipofectamine 3000 Reagent (Invitrogen).

Protein extraction and western blot analysis. Total proteins were lysed with RIPA lysis buffer with proteinase inhibitor (both from Beyotime, Shanghai, China) extracted from cancer cells. Proteins with the same quality were separated by sodium dodecyl sulfate-polyacrylamide gel electrophoresis (SDS-PAGE) and transferred onto a PVDF membrane (Bio-Rad Laboratories, Inc., Hercules, CA, USA). The membrane were incubated overnight at $4^{\circ} \mathrm{C}$ with rabbit anti-USP39 polyclonal antibody (dilution, 1:1,000, cat. no. U0385; Sigma-Aldrich), with anti-GAPDH mouse monoclonal antibody (dilution 1:2,000, cat. no. G8795; Sigma-Aldrich) as internal control. After washed the extra antibody with TBST, the membranes were incubated with rabbit secondary antibody (dilution 1:5,000, cat. no. sc-362280; Santa Cruz Biotechnology, Inc., Dallas, TX, USA) containing horseradish peroxidase-conjugated for $2 \mathrm{~h}$ at room temperature. ECL Western Blotting Detection System (BestBio, Beijing, China) was applying to perform the interest proteins (CCNG1 and GAPDH) and visualized on Bio-Rad Gel Doc XR instrument (Bio-Rad Laboratories, Inc.).

Cell proliferation assay. Cells were seeded in 96-well plates and 3-(4,5-dimethyl-2-thiazolyl)-2,5-diphenyl-2H-tetrazolium bromide (MTT; Santa Cruz Biotechnology, Inc.) was dissolved in phosphate-buffered saline (Biotech, Jiangsu, China) before experiment. MTT and dimethyl sulfoxide (DMSO) solutions were utilized to detect cell proliferative activity. After the cells were cultured 1, 2, 3 or 4 days, $10 \mu 1$ MTT solution was added into each well. Then, $150 \mu \mathrm{l}$ DMSO was added to destroy the cells after incubation at $37^{\circ} \mathrm{C}$ for approximately $4 \mathrm{~h}$. Finally, after agitation for $10 \mathrm{~min}$, absorbance was measured at $490 \mathrm{~nm}$ on a microplate reader (BioTek Instruments, Inc., Winooski, VT, USA).

Plasmid construction and luciferase reporter assay. TargetScan (http://www.targetscan.org/vert_71/) was used to predict potential target genes of miR-133a and identified USP39 as a potential target. Firstly, we cloned miR-133a mimic into pmirGlo vector and inserted the target sequences on USP39 into pcDNA3.1 plasmid vector (pcDNA3.1-USP39-WT). Secondly, after mutation (QuikChange Multi Site-Directed Mutagenesis kit; Santa Clara, CA, USA) the target sequences used were from $5^{\prime}-\ldots$ A CCAGCA...-3' to 5'-...UAUCGCA...-3', and the mutation fragment was inserted into pcDNA3.1 plasmid vector (pcDNA3.1-USP39-MUT).

Luciferase reporter activity was measured after co-transfection with miR-133a mimic or negative control and pcDNA3.1-USP39-WT or pcDNA3.1o-USP39-MUT into gastric cancer cells HGC-27 and MGC-803. The experiment kit used was the Dual-Luciferase ${ }^{\circledR}$ Reporter Assay System (Promega, Madison, WI, USA) and Renilla luciferase was used as normalization.

Statistical analysis. Statistical analyses were presented as the mean \pm standard deviation using SPSS19.0 software 
Table I. miR-133a expression and clinicopathological characteristics in 53 paired gastric cancer.

\begin{tabular}{|c|c|c|c|c|}
\hline \multirow{2}{*}{$\begin{array}{l}\text { Clinicopathological } \\
\text { characteristics }\end{array}$} & \multirow[b]{2}{*}{ Cases $(n=53)$} & \multicolumn{2}{|c|}{ miR-133a expression } & \multirow[b]{2}{*}{ P-value } \\
\hline & & High $(\%)$ & Low $(\%)$ & \\
\hline \multicolumn{5}{|l|}{ Sex } \\
\hline Male & 30 & $14(46.7)$ & $16(53.3)$ & \multirow[t]{2}{*}{0.511} \\
\hline Female & 23 & $13(56.5)$ & $12(43.5)$ & \\
\hline \multicolumn{5}{|l|}{ Age (years) } \\
\hline$\leq 60$ & 21 & $13(61.9)$ & $8(38.1)$ & \multirow[t]{2}{*}{0.082} \\
\hline$>60$ & 32 & $12(37.5)$ & $20(62.5)$ & \\
\hline \multicolumn{5}{|l|}{ Tumor size (mm) } \\
\hline$\leq 5.0$ & 23 & $15(65.2)$ & $8(34.8)$ & \multirow[t]{2}{*}{$0.021^{\mathrm{a}}$} \\
\hline$>5.0$ & 30 & $10(33.3)$ & $20(66.7)$ & \\
\hline \multicolumn{5}{|l|}{ TNM stage } \\
\hline I-II & 24 & $15(62.5)$ & $9(37.5)$ & \multirow[t]{2}{*}{$0.042^{\mathrm{a}}$} \\
\hline III-IV & 29 & $10(34.5)$ & $19(65.5)$ & \\
\hline \multicolumn{5}{|l|}{ Local invasion } \\
\hline $\mathrm{T} 1-\mathrm{T} 2$ & 25 & $15(60.0)$ & $10(40.0)$ & \multirow[t]{2}{*}{0.077} \\
\hline $\mathrm{T} 3-\mathrm{T} 4$ & 28 & $10(35.7)$ & $18(64.3)$ & \\
\hline \multicolumn{5}{|c|}{ Lymph-node metastasis } \\
\hline $0-2$ & 29 & $18(62.1)$ & $11(37.9)$ & \multirow[t]{2}{*}{$0.017^{\mathrm{a}}$} \\
\hline$>2$ & 24 & $7(29.2)$ & $17(70.8)$ & \\
\hline \multicolumn{5}{|l|}{ Ki-67 } \\
\hline$<14 \%$ & 15 & $10(66.7)$ & $5(33.3)$ & \multirow[t]{2}{*}{0.074} \\
\hline$\geq 14 \%$ & 38 & $15(39.5)$ & $23(60.5)$ & \\
\hline \multicolumn{5}{|l|}{ USP39 } \\
\hline Negative & 27 & $17(63.0)$ & $10(37.0)$ & \multirow[t]{2}{*}{$0.019^{\mathrm{a}}$} \\
\hline Positive & 26 & $8(30.8)$ & $18(69.2)$ & \\
\hline
\end{tabular}

${ }^{\text {aP}} \mathrm{P}$-values are calculated with $\chi^{2}$ test. miR, microRNA.

(SPSS, Inc., Chicago, IL, USA). The Student's t-test or ANOVA and Scheffe test were used to perform the statistical analysis. Pearson's $\chi^{2}$ test was used to test the correlation between miR-133a and USP39 and clinicopathologic characteristics of gastric carcinoma. In addition, the Kaplan-Meier method with log-rank test was used for analyzing survival. $\mathrm{P}<0.05$ was considered to indicate a statistically significant difference.

\section{Results}

Low expression of miR-133a in gastric cancer and suppressed cell proliferation. The expression of miR-133a in gastric cancer and corresponding paracancerous tissues were measured by RT-PCR. Results showed that, the average expression level of miR-133a was downregulated in gastric cancer compared with paracancerous tissues $(\mathrm{P}<0.001)$ (Fig. 1A). Further analysis revealed that miR-133a expression level was downregulated in gastric cancer cells HGC-27 and MGC-803 and normal cell GES-1 on contrast to normal gastric cell (both $\mathrm{P}<0.001)$ (Fig. 1B).

On the other hand, we assumed that miR-133a played an important role on the proliferation of glioma, thus the effect of miR-133a on glioma proliferation was detected. For the sake of testing the impact of miR-133a on proliferation, we utilized miR-133a mimic to overexpress miR-133a in gastric cancer cell lines HGC-27 and MGC-803 (both $\mathrm{P}<0.001$ ), as shown in Fig. 1C. Subsequently, we measured cell proliferative ability and found that overexpressed miR-133a resulted in decreased proliferation ability both in HGC-27 $(\mathrm{P}<0.001)$ and MGC-803 ( $\mathrm{P}<0.001)$ (Fig. 1D).

USP39 was overexpressed and promoted cell proliferation in gastric cancer. The expression of USP39 in gastric cancer and paracancerous tissues were also measured by RT-PCR. The result was USP39 upregulated in gastric cancer on contrast to paracancerous tissues $(\mathrm{P}<0.001)$ (Fig. 2A). Furthermore, we detected that USP39 expression in gastric cancer cell lines HGC-27 and MGC-803 and normal gastric cell GES-1 respectively. It was highly expressed in gastric cancer cells compared with normal cells $(\mathrm{P}<0.001$ both in HGC-27 and MGC-803) (Fig. 2B).

To test the effect of USP39 on proliferation, we used siRNA-USP39 to interfere with USP39 expression and the results $(\mathrm{P}<0.001)$ were measured using $\mathrm{RT}-\mathrm{PCR}$, as shown in Fig. 2C. Following, we measured cell proliferative ability and found that the proliferative ability was increased both in 
A

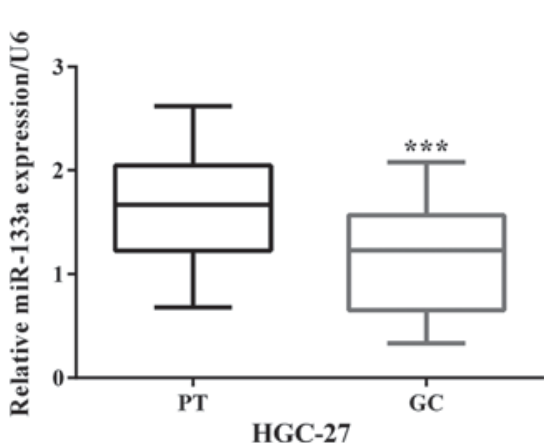

C

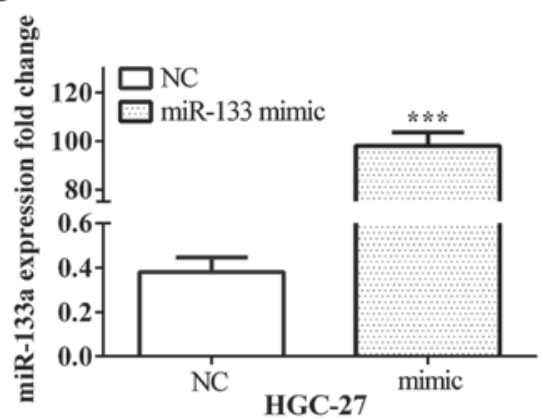

D

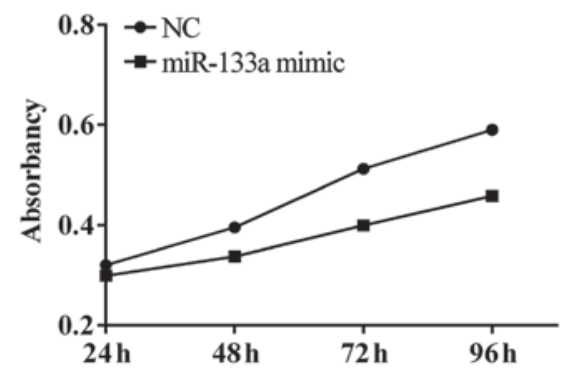

B
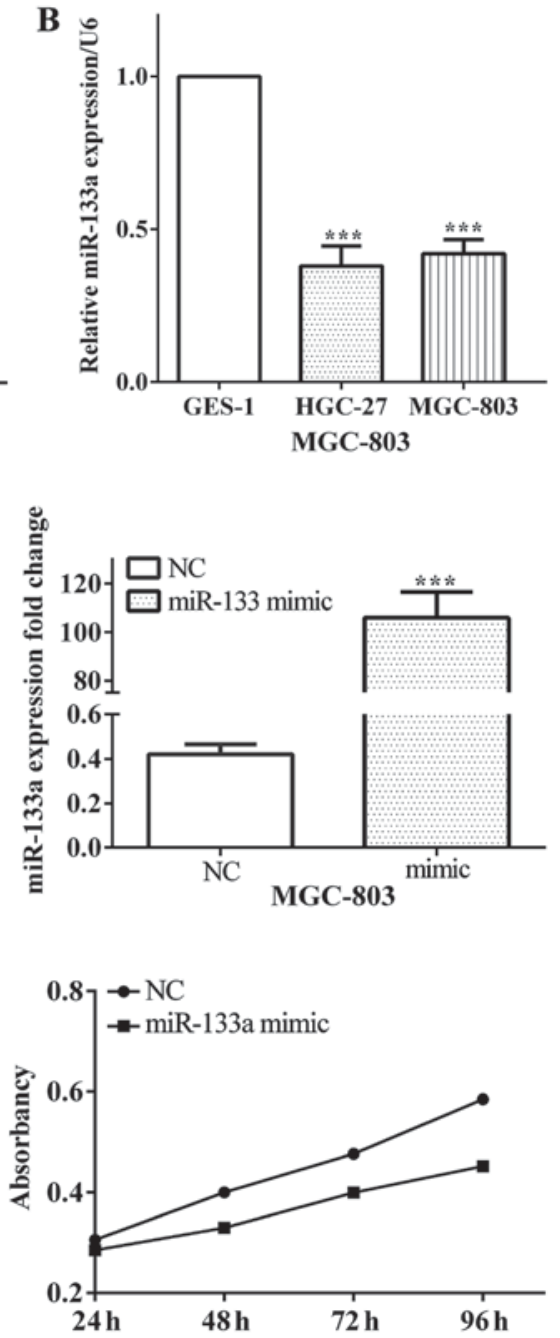

Figure 1. miR-133a was lowly expressed in gastric cancer and suppressed cell proliferation. (A) miR-133a was lowly expressed in gastric cancer tissues compared with paracancerous tissues. (B) Low expression of miR-133a in gastric cancer cells HGC-27 and MGC-803 compared with normal gastric cell GES-1. (C) miR-133a was overexpressed by transfected miR-133a mimic. (D) miR-133a overexpression inhibits gastric cell proliferation. ${ }^{* * * *} \mathrm{P}<0.001$. miR, microRNA; PT, paracancerous tissues; GC, gastric cancer tissues; NC, negative control.

HGC-27 $(\mathrm{P}=0.0002)$ and MGC-803 $(\mathrm{P}<0.001)$ when interfered with USP39 (Fig. 2D).

USP39 is a direct target of $\mathrm{miR}-133 \mathrm{a}$ and partially reversed function of $m i R-133 a$. We predicted that USP39 was a potential downstream target of miR-133a using online software TargetScan with binding site of USP39 was at its 3'UTR located at 79 to 85 . The mutated target sequences from 5'-...AUAAUGAUGAAACCAACCAGCAG...-3' to 5'-...AU AAUGAUGAAACCAUAUCGCAC...-3', and inserted the wild-type (pcDNA3.1-USP39-WT) and mutant type (pcDNA3.1-USP39-MUT) fragment into pcDNA3.1 plasmid vector in prior to the performance of the luciferase reporter assay (Fig. 3A). In addition, we compared the expression of miR-133a and USP39, and found a negative correlation between miR-133a and USP39 with $\mathrm{r}=-0.8146$ and $\mathrm{P}<0.001$ (Fig. 3B).

To confirm USP39 was directly suppressed by miR-133a, we carried out a luciferase reporter assay. Following the protocol, we co-transfected pmirGlo-miR-133a mimic or negative control and pcDNA3.1-USP39-WT or pcDNA3.1-USP39-MUT into the gastric cancer cell lines HGC-27 and MGC-803, respectively, and measured the luciferase reporter activity. Co-transfection experiments in HGC-27 and MGC-803 cells showed that miR-133a significantly decreased the luciferase activity of pcDNA3.1-USP39-WT 3'UTR (both $\mathrm{P}<0.001$ ); however, this was not observed in pcDNA3.1-USP39-MUT 3'UTR ( $\mathrm{P}=0.486$ and $\mathrm{P}=0.190$, respectively) (Fig. 3C).

Furthermore, when miR-133a was overexpressed by transfected miR-133a mimic, the expression of USP39 was decreased in HGC-27 ( $\mathrm{P}=0.0003)$ and MGC-803 $(\mathrm{P}=0.0002)$ (Fig. 4A). The expression of USP39 was reduced when transfected with miR-133a mimic $(\mathrm{P}=0.0007)$, which was re-expressed via overexpressed USP39 ( $\mathrm{P}=0.0179)$. USP39 overexpression deprived miR-133a inhibitor-mediated suppression of cell proliferation, suggesting that USP39 is involved in miR-133a-mediated biological role in gastric cancer cell HGC-27 ( $\mathrm{P}<0.0001)$ (Fig. 4C). Therefore, depletion of USP39 reversed the partial function of miR-133a.

miR-133a lowly expressed or USP39 overexpressed predicted poor prognosis. We divided 53 gastric cancer patients into the high [miR-133a(+)] and low [miR-133a(-)] expression groups according to miR-133a expression level, with 25 and 28 patients, 
A
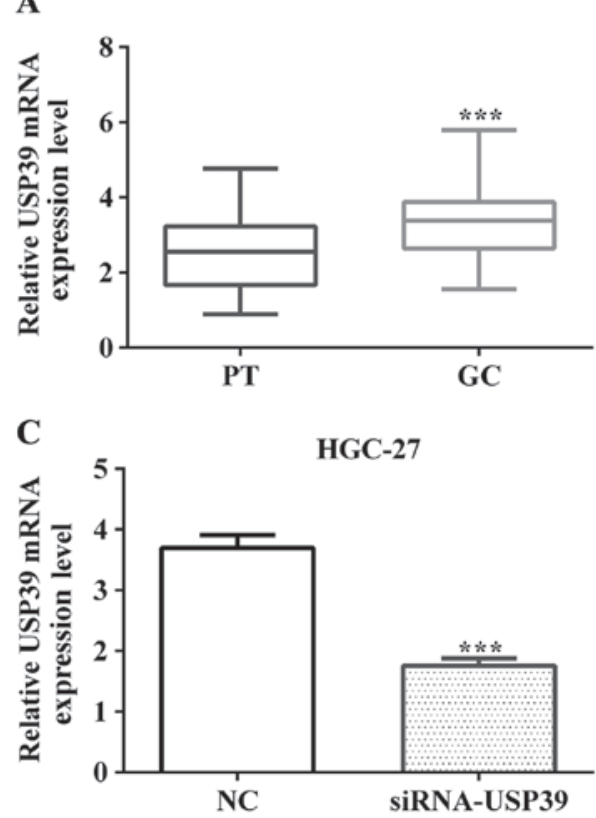

D

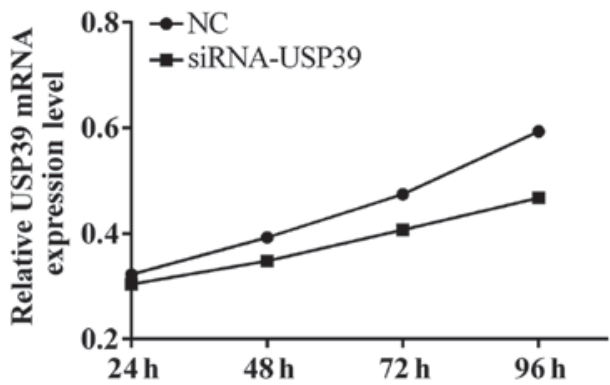

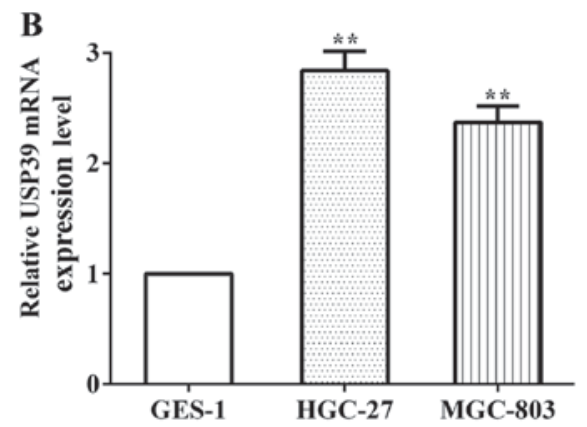

MGC-803

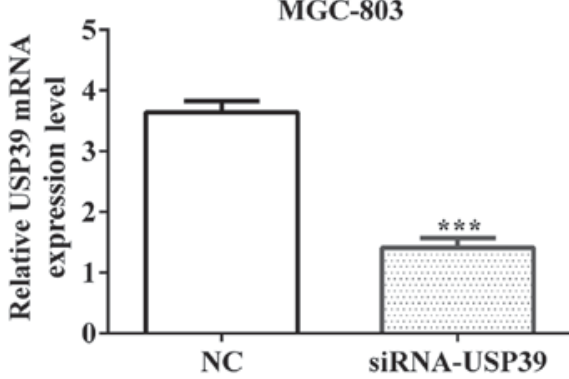

MGC-803

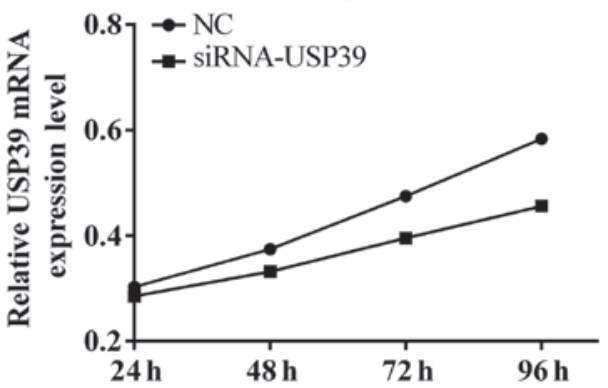

Figure 2. USP39 was overexpressed and promoted cell proliferation in gastric cancer. (A and B) USP39 was overexpressed in gastric cancer tissues and cells compared with paracancerous tissues and normal gastric cell. (C) Interference with USP39 expression by siRNA. (D) USP39 low expression suppressed gastric cell proliferation. ${ }^{* *} \mathrm{P}<0.01,{ }^{* * *} \mathrm{P}<0.001$. USP39, ubiquitin specific protease 39 ; mRNA, messenger RNA; siRNA, small interfering RNA; PT, paracancerous tissues; GC, gastric cancer tissues; NC, negative control.

A

\begin{tabular}{|c|c|}
\hline \multicolumn{2}{|c|}{ Site: $79-85$ of USP39 } \\
\hline 3'UTR WT & 5'-...AUAAUGAUGAAACCAACCAGCAG....3' \\
\hline hsa-miR-133a & $3^{\prime}-\ldots$ UAAACCAAGGUAAAAUGGUCGUA....5' \\
\hline 3'UTR MUT & $5 '-\ldots$ AUAAUGAUGAAACCAUAUCGCAC...3 \\
\hline
\end{tabular}

C

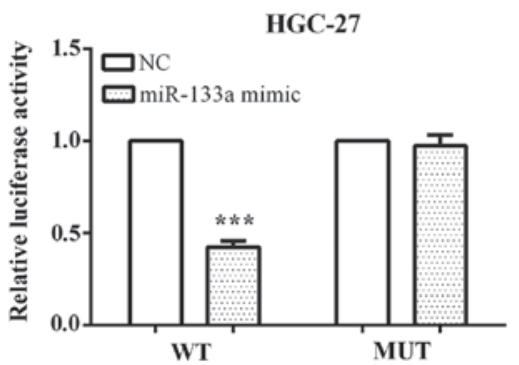

B

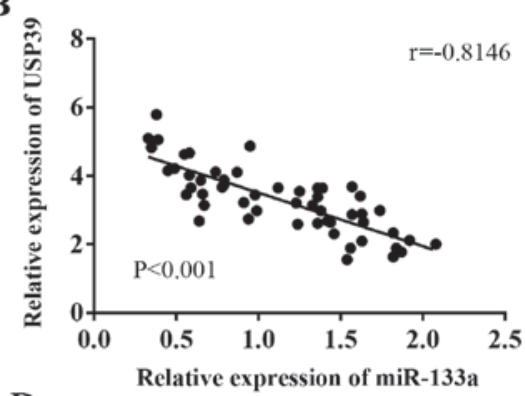

D

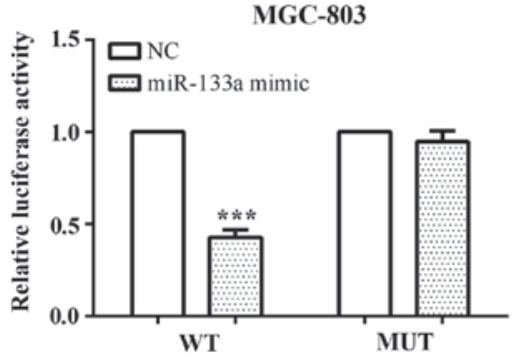

Figure 3. USP39 was a direct target of miR-133a and mediated by miR-133a. (A) The binding sites of USP39 and miR-133a, and the mutation of USP39. (B) Negative correlation between the expression of miR-133a and USP39. (C) miR-133a targeted the wild-type of USP39 but not the mutant 3'UTR. (D) miR-133a downregulated the expression of USP39 detected by RT-PCR and WB. ${ }^{* * *} \mathrm{P}<0.001$. USP39, ubiquitin specific protease 39; miR, microRNA; 3'UTR, 3'-untranslated regions; WT, wild-type of USP39 3'UTR; MUT, mutant of USP39 3'UTR; NC, negative control. 
$\mathbf{A}$

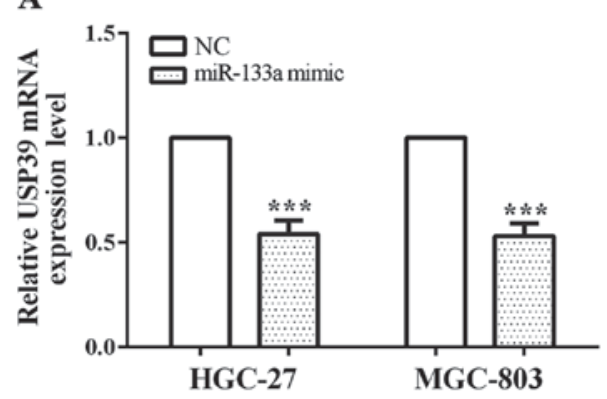

C

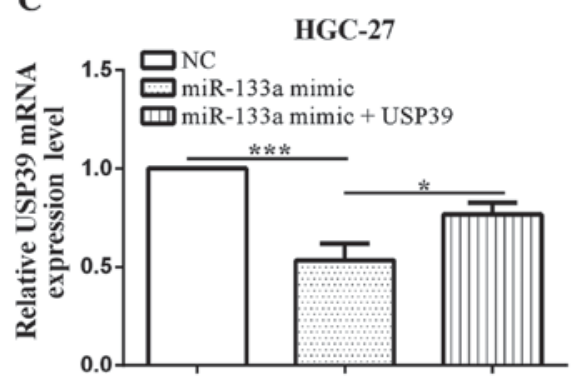

B

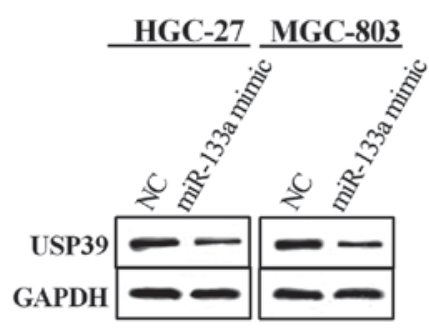

D

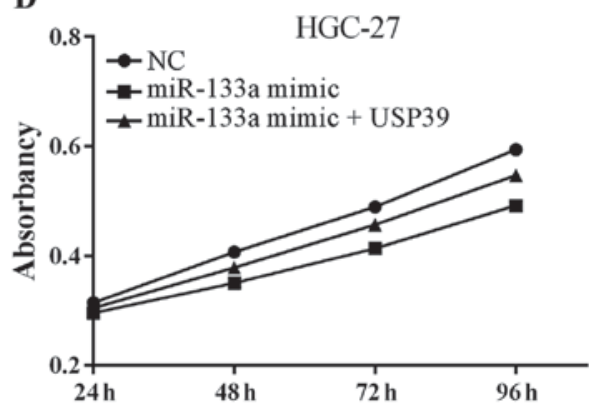

Figure 4. USP39 reversed the partial function of miR-133a. (A and B) RT-qPCR and western blot analysis of the USP39 expression level in HGC-27 and MGC-803 cells after transfection with miR-133a mimic. The results confirmed the transfection efficiency. (C) USP39 expression was reduced following transfection with miR-133a mimic, which was re-expressed via overexpression of USP39. (D) USP39 overexpression deprived miR-133a inhibitor-mediated suppression of cell proliferation. ${ }^{*} \mathrm{P}<0.05,{ }^{* * *} \mathrm{P}<0.001$. USP39, ubiquitin specific protease 39; NC, negative control; miR, microRNA; mRNA, messenger RNA.

A

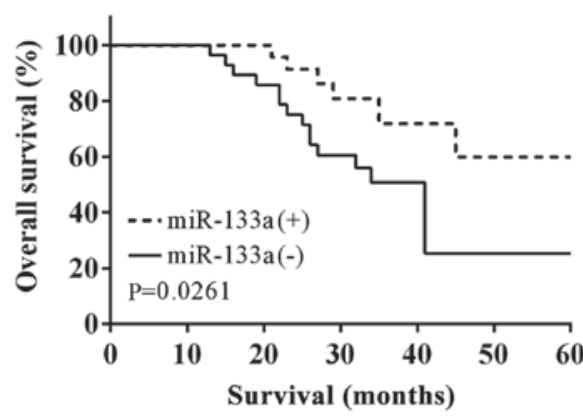

C

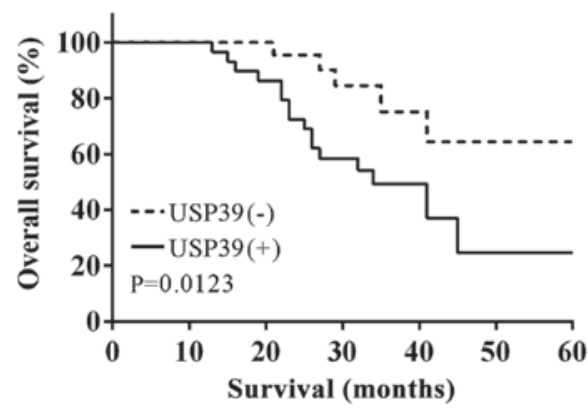

B

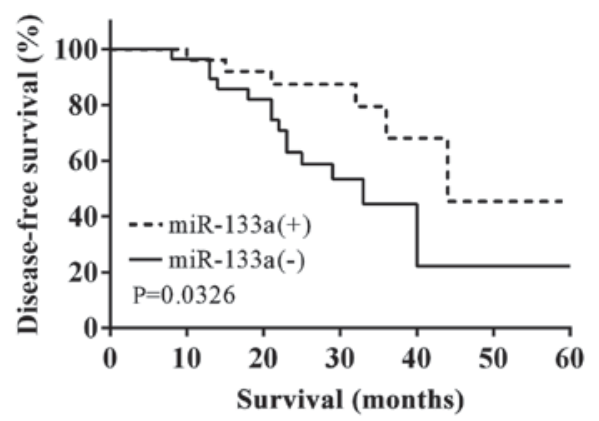

D

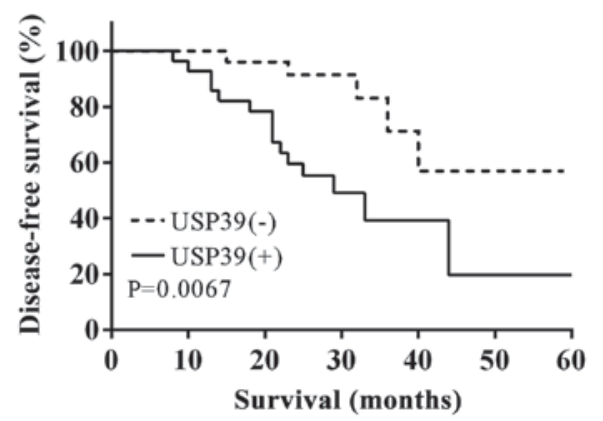

Figure 5. Low expression of miR-133a or USP39 overexpression predicted poor prognosis. (A and B) OS and DFS were calculated between patients according to the expression of miR-133a. (C and D) OS and DFS were calculated on the basis of the expression of USP39. miR, microRNA; USP39, ubiquitin-specific protease 39; OS, overall survival; DFS, disease-free survival.

respectively. Furthemore, 53 patients were separated into two groups on the basis of sex, age, tumor size, TNM stage, local invasion, lymph-node metastasis, Ki-67 and USP39, respectively, and the detailed grouping is shown in Table I. The expression of miR-133a had a negative correlation with tumor size $(\mathrm{P}=0.021)$, TNM stage $(\mathrm{P}=0.042)$, lymph-node metastasis $(\mathrm{P}=0.017)$ and USP39 $(\mathrm{P}=0.019)$. However, there was a tendency for the expression to be associated with age $(\mathrm{P}=0.082)$, local invasion $(\mathrm{P}=0.077)$ and $\mathrm{Ki}-67(\mathrm{P}=0.074)$. No association was identified between miR-133a with sex $(\mathrm{P}=0.511)$ (Table I). 
We calculated the OS and DFS with the aid of Kaplan-Meier in accordance with the expression of miR-133a, and found that the OS and DFS in the miR-133a(+) group was significantly higher than that in the miR-133a(-) group (log-rank test, $\mathrm{P}=0.0261$ and $\mathrm{P}=0.0326$ ) (Fig. $5 \mathrm{~A}$ and $\mathrm{B}$ ). In addition, we measured OS and DFS according to USP39 expression, and the opposite results were obtained, i.e., OS and DFS were lower when USP39 overexpression compared with a low expression (log-rank test, $\mathrm{P}=0.0123$ and $\mathrm{P}=0.0067$ ) (Fig. 5C and D).

\section{Discussion}

Gastric cancer mortality is ranked second only to lung cancer, and generally diagnosed at an advance stage. The disease is prone to metastasis and recurrence. Although cancer treatments have been improved in recent years, the outcomes of patients with GC remain unsatisfactory $(1,2)$. Thus, identifying new biomarkers for GC effective therapeutics is urgent. miRNAs typically bind to the mRNA 3'-UTR of its target gene or directly cut the mRNA at a post-transcriptional level, thereby inhibiting the expression of the target gene $(5,6)$. miR-133a has been reported to be an antioncogene, which was correlated with cell development, proliferation and prognosis in colon cancer, oral squamous cell carcinoma and gastric cancer $(14,16,27)$. In the present study, we verified that USP39 was a direct target of miR-133a in gastric cancer cell lines GC-27 and MGC-803. A high expression of USP39 was also identified in gastric cancer tissues and cell lines, while miR-133a was lowly expressed compared with paracancerous tissues and normal cells. Considering these results, we strongly believe that the impact of miR-133a on proliferation may be through direct inhibition of USP39. Gong et al indicated that miR-133a suppressed gastric cancer cell growth, migration and invasion and induced cell apoptosis in vitro and inhibited gastric cancer growth in vivo, thus obtaining the result that miR-133a was a tumor suppressor in vitro and in vivo (17). Other findings have shown that miR-133a inhibited gastric cancer cell proliferative and invasive ability and promoted apoptosis (16). Although miR-133a is considered a tumor suppressor factor in gastric cancer, we suggest miR-133a directly targets USP39 and affects proliferation by regulating USP39. In addition, to the best of our knowledge, this is the first study to focus on the impact of miR-133a and USP39 on the survival of gastric cancer patients.

The effect of miR-133a on cell proliferation, invasion and cell cycle have been studied previously, although via different cell lines and biomarkers $(12,16,17,28)$. Li et al have shown that miR-133a inhibits gastric cancer cell proliferation by downregulating ERBB2 expression (12). Similar findings were reported by Lai et al and Gong et al, who showed that miR-133a inhibited proliferation, migration and invasion, and induced apoptosis in gastric cancer cells $(16,17)$. Our findings were consistent with previous findings $(12,16,17)$, as we have shown that overexpression of miR-133a may inhibit proliferation by directly targeting USP39 in gastric cancer cell lines HGC-27 and MGC-803. In addition, we identified that miR-133a downregulation and/or USP39 upregulation predict poor prognosis.

In the present study, it was identified that USP39 was involved in influencing miR-133a on HGC-27 and MGC-803 cell proliferation. USP39 was a kind of DUBs without ubiquitin protease activity, which played an important role in RNA splicing and necessary to maintain spindle checkpoint (19-22). USP39 is essential for mitotic spindle checkpoint integrity, and a decrease of USP39 could suppress cell division (22). Furthermore, USP39 overexpression has been reported in colorectal cancer, lung cancer, melanoma, pancreatic cancer and osteosarcoma cancer (18,23-26). In addition, it has been reported that overexpression of MGC-803 cells and knockdown of USP39 may inhibit MGC-803 cell proliferation and induce cell cycle arrest (29). Furthermore, Wen et al reported that USP39 overexpression reduced patient survival times in prostate cancer (30). In the present study, USP39 was over0expressed in gastric cancer tissues and cell lines HGC-27 and MGC-803 compared with paracancerous tissues and normal gastric cells. USP39 overexpression promotes cell proliferation and predicts poor prognosis, which is consistent with present research $(29,30)$. Therefore, the transfection of miR-133a mimic inhibits USP39 expression, thereby causing cell proliferation. Thus, miR-133a and USP39 were associated with the prognosis of patients. Due to the limitation of experimental conditions, no flow cytometry was carried out in our laboratory. Additionally, we did not conduct more experiments to verify the effects of miR-133a and USP39 on cell proliferation.

In addition, the expression of USP39 has been reported to be downregulated by miR-133a and was negatively correlated with miR-133a expression in pancreatic cancer (18). Similarly, in this study, we demonstrated that USP39 was a direct target of miR-133a and mediated by miR-133a in gastric cancer cell lines. However, there was no further mechanism explaining how miR-133a affected GC proliferation through USP39. This should be considered as a limitation of our study. The role of USP39 in the molecular mechanisms involving in migration and invasion of GC will be studied in further research.

In conclusion, we have indicated that miR-133a acts as a tumor suppressor in GC by inhibiting cancer proliferation. Furthermore, we demonstrated that miR-133a has an inverse correlation with USP39 and directly targets it by binding to its 3'UTR. This novelty of miR-133a may offer a promising therapeutic target for the treatment of GC.

\section{Acknowledgements}

Not applicable.

\section{Funding}

No funding was received.

\section{Availability of data and materials}

The datasets used and/or analyzed during the present study are available from the corresponding author on reasonable request.

\section{Authors' contributions}

$\mathrm{XD}$ and HS contributed equally to this study and share first authorship. FJ as the third author analysed and prepared the manuscrit. HL and GS as the fourth and fifth authors analysed the data and wrote the manuscript; LF as the corresponding 
author contributed to the conception of the study. All authors read and approved the final manuscript.

\section{Ethics approval and consent to participate}

Informed consent to use the specimens in this study was obtained from the patients. The study was approved by the Ethics Committee of Jining First People's Hospital (Jining, China).

\section{Consent for publication}

Not applicable.

\section{Competing interests}

The authors declare that they have no competing interests.

\section{References}

1. Piazuelo MB and Correa P: Gastric cancer: Overview. Colomb Med (Cali) 44: 192-201, 2013.

2. Jemal A, Bray F, Center MM, Ferlay J, Ward E and Forman D: Global cancer statistics. CA Cancer J Clin 61: 69-90, 2011.

3. Chen Y, Lin WS, Zhu WF, Lin J, Zhou ZF, Huang CZ, Chen G, Shi Y, Guo ZQ and Ye YB: Tumor MICA status predicts the efficacy of immunotherapy with cytokine-induced killer cells for patients with gastric cancer. Immunol Res 64: 251-259, 2016.

4. Choi YY, Noh SH and Cheong JH: Evolution of gastric cancer treatment: From the golden age of surgery to an era of precision medicine. Yonsei Med J 56: 1177-1185, 2015.

5. Christodoulatos GS and Dalamaga M: Micro-RNAs as clinical biomarkers and therapeutic targets in breast cancer: Quo vadis? World J Clin Oncol 5: 71-81, 2014.

6. Lauressergues D, Couzigou JM, Clemente HS, Martinez Y, Dunand C, Bécard G and Combier JP: Primary transcripts of microRNAs encode regulatory peptides. Nature 520: 90-93, 2015.

7. Voinnet O: Origin, biogenesis, and activity of plant microRNAs. Cell 136: 669-687, 2009.

8. Di Giacomo G, Koss M, Capellini TD, Brendolan A, Pöpperl H and Selleri L: Spatio-temporal expression of $\mathrm{Pbx} 3$ during mouse organogenesis. Gene Expr Patterns 6: 747-757, 2006.

9. Lichtenauer UD, Duchniewicz M, Kolanczyk M, Hoeflich A, Hahner S, Else T, Bicknell AB, Zemojtel T, Stallings NR, Schulte DM, et al: Pre-B-cell transcription factor 1 and steroidogenic factor 1 synergistically regulate adrenocortical growth and steroidogenesis. Endocrinology 148: 693-704, 2007.

10. Fujiwara T, Katsuda T, Hagiwara K, Kosaka N, Yoshioka Y, Takahashi RU, Takeshita F, Kubota D, Kondo T, Ichikawa H, et al: Clinical relevance and therapeutic significance of microRNA-133a expression profiles and functions in malignant osteosarcoma-initiating cells. Stem Cells 32: 959-973, 2014.

11. Guo J, Xia B, Meng F and Lou G: miR-133a suppresses ovarian cancer cell proliferation by directly targeting insulin-like growth factor 1 receptor. Tumour Biol 35: 1557-1564, 2014.

12. Li C, Li X, Gao S, Li C and Ma L: MicroRNA-133a inhibits proliferation of gastric cancer cells by downregulating ERBB2 expression. Oncol Res 25: 1169-1176, 2017.

13. Wang LK, Hsiao TH, Hong TM, Chen HY, Kao SH, Wang WL, Yu SL, Lin CW and Yang PC: MicroRNA-133a suppresses multiple oncogenic membrane receptors and cell invasion in non-small cell lung carcinoma. PLoS One 9: e96765, 2014.
14. He B, Lin X, Tian F, Yu W and Qiao B: MiR-133a-3p inhibits oral squamous cell carcinoma (OSCC) proliferation and invasion by suppressing COL1A1. J Cell Biochem 119: 338-346, 2018

15. Li W, Chen A, Xiong L, Chen T, Tao F, Lu Y, He Q, Zhao L, Ou R and $\mathrm{Xu}$ Y: miR-133a acts as a tumor suppressor in colorectal cancer by targeting eIF4A1. Tumour Biol 39: 1010428317698389, 2017.

16. Lai C, Chen Z and Li R: MicroRNA-133a inhibits proliferation and invasion, and induces apoptosis in gastric carcinoma cells via targeting fascin actin-bundling protein 1 . Mol Med Rep 12: 1473-1478, 2015

17. Gong Y, Ren J, Liu K and Tang LM: Tumor suppressor role of miR-133a in gastric cancer by repressing IGF1R. World J Gastroenterol 21: 2949-2958, 2015.

18. Cai J, Liu T, Huang P, Yan W, Guo C, Xiong L and Liu A: USP39, a direct target of microRNA-133a, promotes progression of pancreatic cancer via the AKT pathway. Biochem Biophys Res Commun 486: 184-190, 2017.

19. Reyes-Turcu FE, Ventii KH and Wilkinson KD: Regulation and cellular roles of ubiquitin-specific deubiquitinating enzymes. Annu Rev Biochem 78: 363-397, 2009.

20. Lygerou Z, Christophides G and Séraphin B: A novel genetic screen for snRNP assembly factors in yeast identifies a conserved protein, Sad1p, also required for pre-mRNA splicing. Mol Cell Biol 19: 2008-2020, 1999.

21. Clague MJ, Barsukov I, Coulson JM, Liu H, Rigden DJ and Urbé S: Deubiquitylases from genes to organism. Physiol Rev 93: 1289-1315, 2013

22. van Leuken RJ, Luna-Vargas MP, Sixma TK, Wolthuis RM and Medema RH: Usp39 is essential for mitotic spindle checkpoint integrity and controls mRNA-levels of aurora B. Cell Cycle 7: 2710-2719, 2008.

23. Yuan X, Sun X, Shi X, Wang H, Wu G, Jiang C, Yu D, Zhang W, Xue B and Ding Y: USP39 promotes colorectal cancer growth and metastasis through the $\mathrm{Wnt} / \beta$-catenin pathway. Oncol Rep 37: 2398-2404, 2017.

24. Lin Z, Xiong L and Lin Q: Ubiquitin-specific protease 39 is overexpressed in human lung cancer and promotes tumor cell proliferation in vitro. Mol Cell Biochem 422: 97-107, 2016.

25. Zhao Y, Zhang B, Lei Y, Sun J, Zhang Y, Yang S and Zhang X: Knockdown of USP39 induces cell cycle arrest and apoptosis in melanoma. Tumour Biol 37: 13167-13176, 2016.

26. Gan Z, Han K, Lin S, Hu H, Shen Z and Min D: Knockdown of ubiquitin-specific peptidase 39 inhibited the growth of osteosarcoma cells and induced apoptosis in vitro. Biol Res 50: 15, 2017.

27. Li W, Chen A, Xiong L, Chen T, Tao F, Lu Y, He Q, Zhao L, Ou R and $\mathrm{Xu} \mathrm{Y:} \mathrm{miR-133a} \mathrm{acts} \mathrm{as} \mathrm{a} \mathrm{tumor} \mathrm{suppressor} \mathrm{in} \mathrm{colorectal}$ cancer by targeting eIF4A1. Tumour Biol 39: 1010428317698389 , 2017.

28. Qiu T, Zhou X, Wang J, Du Y, Xu J, Huang Z, Zhu W, Shu Y and Liu P: MiR-145, miR-133a and miR-133b inhibit proliferation, migration, invasion and cell cycle progression via targeting transcription factor Sp1 in gastric cancer. FEBS Lett 588: 1168-1177, 2014.

29. Wang X, Yu Q, Huang L and Yu P: Lentivirus-mediated inhibition of USP39 suppresses the growth of gastric cancer cells via PARP activation. Mol Med Rep 14: 301-306, 2016.

30. Wen D, Xu Z, Xia L, Liu X, Tu Y, Lei H, Wang W, Wang T, Song L, Ma C, et al: Important role of SUMOylation of Spliceosome factors in prostate cancer cells. J Proteome Res 13: 3571-3582, 2014

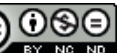

This work is licensed under a Creative Commons Attribution-NonCommercial-NoDerivatives 4.0 International (CC BY-NC-ND 4.0) License. 